\title{
Liposome-Based Delivery Systems in Plant Polysaccharides
}

\author{
Meiwan Chen, ${ }^{1}$ Yanfang Zhou, ${ }^{2}$ Jingjing Huang, ${ }^{3}$ Ping Zhu, ${ }^{3}$ \\ Xinsheng Peng, ${ }^{2}$ and Yitao Wang ${ }^{1}$ \\ ${ }^{1}$ State Key Laboratory of Quality Research in Chinese Medicine, Institute of Chinese Medical Sciences, University of Macau, \\ Macau 999078, China \\ ${ }^{2}$ School of Pharmaceutical Sciences, Guangdong Medical College, Dongguan 523808, China \\ ${ }^{3}$ Department of Cardiovascular Surgery, Guangdong Cardiovascular Institute, Guangdong General Hospital, \\ Guangdong Academy of Medical Sciences, Guangzhou 510100, China
}

Correspondence should be addressed to Ping Zhu, tanganqier@163.com and Xinsheng Peng, xsgz@yahoo.com.cn

Received 17 January 2012; Revised 11 February 2012; Accepted 29 February 2012

Academic Editor: Ping Xiao

Copyright (C) 2012 Meiwan Chen et al. This is an open access article distributed under the Creative Commons Attribution License, which permits unrestricted use, distribution, and reproduction in any medium, provided the original work is properly cited.

\begin{abstract}
Plant polysaccharides consist of many monosaccharide by $\alpha$ - or $\beta$-glycosidic bond which can be extracted by the water, alcohol, lipophile liquid from a variety of plants including Cordyceps sinensis, astragalus, and mushrooms. Recently, many evidences illustrate that natural plant polysaccharides possess various biological activities including strengthening immunity, lowering blood sugar, regulating lipid metabolism, antioxidation, antiaging, and antitumour. Plant polysaccharides have been widely used in the medical field due to their special features and low toxicity. As an important drug delivery system, liposomes can not only encapsulate small-molecule compound but also big-molecule drug; therefore, they present great promise for the application of plant polysaccharides with unique physical and chemical properties and make remarkable successes. This paper summarized the current progress in plant polysaccharides liposomes, gave an overview on their experiment design method, preparation, and formulation, characterization and quality control, as well as in vivo and in vitro studies. Moreover, the potential application of plant polysaccharides liposomes was prospected as well.
\end{abstract}

\section{Introduction}

Plant polysaccharides extracted from botanical sources (Astragalus, mushrooms, Cordyceps sinensis, and higher plants) have received a growing attention in the biomedical arena in the past decades [1-3]. They exhibit many biological activities including immune regulation, antioxidant and anti-inflammatory actions, antivirus, antitumor, and so on. For example, polysaccharide fractions from tuber of Ophiopogon japonicus were effective indicators of antioxidant and immunomodulation activity, and some polysaccharides extracted from Polyporus albicans mycelia could be potential immunostimulants which can be used in the food and pharmaceutical industry $[4,5]$. A broad range of immunomodulatory activities are effect on the immune functions of regulatory $\mathrm{T}$ cells in postburn sepsis and enhancing host defense responses $[6,7]$, antiherpetic activity by attaching to and/or entry into host cells [8], and reducing oxidative injury and modulating the secretion of cytokine IL-4, IL-5 and IL-17 [3]. Besides, it acts as a potent natural antitumor agent in vivo and in vitro $[9,10]$. Plant polysaccharides have been further optimized into drugs and show clinical efficacy and display a variety of broad spectrum of therapeutic applications such as in the treatment of gastritis and gastroduodenal ulcers [11], being an ideal adjuvants in modern cancer therapy [12]. A chemically sulfated polysaccharide derived from Grifola frondosa mycelia could accelerate the antitumor activity in cancer treatment [13]. Furthermore, it is accompanied by relatively low toxicity [14-16].

Liposomes as a novel platform technology provide an alternative to improve the drug delivery, which composed of a flexible bilayer and surrounded by an aqueous core domain $[17,18]$. Liposome-based delivery systems plays an important role owing to easy preparation, increasing the 
bioavailability, and also offers drug targeting and controlled release [19]. In addition, charged liposomes could be as carriers to enhance the permeation through the skin in the transdermal drug delivery which are administered by the percutaneous route [20].

Recently, it has been reported that liposomes had been employed in the field of plant polysaccharides and made encouraging successes. Liposomes possess unique physical and chemical properties, which not only improve polysaccharide stability, bioavailability, and difficulty in penetration to some cells but also enhance the pharmacodynamic action and induce the target [21]. The development of liposomes of plant polysaccharides is promising, which will be effectively used in the clinical application in the near future. This paper will concentrate on the recent research of plant polysaccharides liposomes, such as their experiment design method, preparation and formulation, characterization, and quality control, as well as in vivo and in vitro studies. We intend to summarize the progress in plant polysaccharides liposomes with the aim to provide reference for research and development of plant polysaccharides.

\section{Experiment Design Method}

To prepare liposomes of plant polysaccharides, lots of factor should be considered such as extraction time of plant polysaccharides, the amount of water added into the plant, excipients to plant polysaccharides ratio, as well as emulsifiable time by ultrasound. Therefore, an ideal experiment design seems important. So far, orthogonal test is widely used to optimize the preparation conditions of plant polysaccharides liposomes. Zuozhen et al. [21] take advantage of orthogonal design to investigate the preparation factor for the cordyceps sinensis Sacc polysaccharide liposomes. The optimum preparation process received was emulsifiable time by ultrasound with $15 \mathrm{~min}$, the plant extracted by water 3 times with $3 \mathrm{~h}$ for each time, 8 times in all the water was as much as the plant [22]. Further study found that preparation conditions of astragalus polysaccharide liposomes optimized by orthogonal design; that is, lecithin to drug ratio was $10: 1$, lecithin to cholesterol ratio was $8: 1$, and ultrasonic time was $20 \mathrm{~min}$. The author concluded that the astragalus polysaccharide liposomes prepared under the optimized conditions had high encapsulation efficiency and drug-loading rate, uniform shape, and particle size, as well as reproducible quality [23].

\section{Preparation Method}

Reverse-phase evaporation method, ether injection, and thin-film dispersion, as well as ethanol injection are the main four methods in preparing plant polysaccharide liposomes. These four methods were compared by some scholars, and the result showed that reverse-phase evaporation method was the sound method to prepare the polysaccharide liposome of Grifola frondosa for high encapsulation efficiency $(20.15 \%)$, round and oval shape, and the mean particle size of $200 \mathrm{~nm}$ [24]. The difference between reverse-rotary evaporation and thin-film dispersion is a big monolayer liposome prepared by reverse rotary and a multiplayer liposome prepared by thin-film dispersion. Reverse-rotary evaporation is a simple operation with general instrument, so it can prepare high-envelopment monolayer liposome. What is more, chloroform infusion combined with the ammonium sulphate gradient method is another common method. It was reported that Polyporus umbellatus polysaccharide longcirculating liposomes prepared by this method can be with high encapsulation efficiency and small particle size [25].

\section{Characterization and Quality Control}

The methods of preparing plant polysaccharides can be divided into three parts. The first one is extraction methods, such as solvent extraction, microwave, and enzyme extraction. The second means is separation and purification methods, covering membrane separation, column chromatography, organic solvent extraction, Sevage method, trifluorotrichloroethane and trichloroacetic acid method, and so on $[26,27]$. The last one is about content determination methods, including carbazole-sulfuric acid colorimetric method, phenol-sulphuric acid method, anthrone sulphuric acid method, and so forth [28]. Therefore, characterization and quality control of plant polysaccharides should be carried out through the process of research and development of plant polysaccharides liposomes. In addition, the physical and chemical properties of plant polysaccharides liposomes should be paied much attention to in the utilization of plant polysaccharide, for example, carrying drug amount, morphology, and entrapment efficiency, as well as particle size distribution.

The carrying drug amount plays an important role in quality control of plant polysaccharide liposome which was usually determined by the UV-Sephadex method and dialysis method. Furthermore, anthrone sulfuric colorimetry is the frequent method to evaluate the content of plant polysaccharides in liposomes. For example, the content of Cordyceps sinensis Sacc polysaccharide in Cordyceps sinensis Sacc polysaccharide liposomal oral liquid was measured by anthrone sulfuric colorimetry was $6.448 \times 10^{-2} \mathrm{~mol} / \mathrm{L}$ [29].

Another important factor which we have to pay more attention to is the entrapment efficiency. Entrapment efficiency is exhibited the capacity of liposomes, to load the polysaccharide. Anion exchange resin was used to separate free plant polysaccharides from the liposome, and UVvisible spectrophotometry or other spectrophotometry was employed to calculate the entrapment efficiency. Liposome entrapment efficiency can be influenced by the dissolution rate and oil-water partition coefficient of plant polysaccharides. The research results showed that the oil-water partition coefficient of ginsenoside liposome was 4.5 , with the entrapment efficiency of $59.06 \%$, while the oil-water partition coefficient of Lycium barbarum polysaccharides was 0.98, with the entrapment efficiency was 47.61\% [30]. Another study showed that the free acidic polysaccharide of Spirulina platensis liposomes (APSP) was separated from the mixture of APSP liposomes preparation by a column of DEAE ion exchange resin, and spectrophotometric method was used to determine the concentration of APSP in solution and the entrapment rate. The results indicated more than $95.4 \%$ 
of the free APSP in liposome preparation, and the average entrapment efficiency of APSP in liposomes preparation was $23.9 \%[31]$.

\section{In Vivo and In Vitro Studies}

Plant polysaccharides are essential for maintaining the physiological metabolism of the body as it possesses a variety of biological activities. They play an important role in regulation of immune, antioxidant, anti-inflammatory, antivirus, antitumor, and so on. At present, studies about the mechanism of plant polysaccharides liposomes were very popular and regulation of immune is the significant bioactivity of polysaccharides $[32,33]$.

To deliver polysaccharide to sites of action, recent technical advances showed that liposomes can fuse with other bilayers and be as specific targeted systems. Thus, it can improve the stability and bioavailability of polysaccharide and enhance its pharmacodynamic action as well [21]. Cai Yun et al. compared the immune regulatory effect of the lentinan liposome and injection in normal mice and found that the effect of lentinan liposome is correlated with dosage and better than lentinan injection statistically. Lentinan liposome indicated potential effect on immune regulation, which could improve the carbon granular clearance, accelerate the proliferation of $\mathrm{T}$ lymphocyte in blood and spleen, as well as enhance the hemolysin production [34]. Recent investigation demonstrated that multilamellar liposomes of laminarin have fully reversible aggregation, while small unilamellar liposomes are with irreversible fusion in the main process [35]. Additionally, the effect of Cordyceps polysaccharide liposomes (CPL) on the expression of transforming factor $\beta 1$ messenger RNA in hepatic tissues and its inhibitive function on the development of immune hepatic fibrosis induced using concanavalin $\mathrm{A}$ (Con $\mathrm{A}$ ) in $\mathrm{BALB} / \mathrm{c}$ mice was measured by Li Hongjun et al. They found that serum levels of alanine aminotransferase (ALT), aspartic acid aminotransferase (AST), serum levels of albumin, hyaluronic acid (HA), procollagen III(PC III) and collagen IV(IVC) in CPL treated groups were obviously decreased as compared to those in Con A group, while the relative expression levels of TGF- $\beta 1 \mathrm{mRNA}$ are significantly statistic lower and dosage dependent [36].

Liposome-based delivery systems are used frequently not only in the process of research and development but also in therapeutic applications [37]. So far, cordyceps sinensis liposome oral liquid is one of good examples. The effect of the cordyceps sinensis liposome oral liquid on the cell immunity of chronic hepatitis B patients in clinical therapy showed that the peripheral blood monnouclear cells, $\mathrm{CD}_{4}{ }^{+} \mathrm{T}$ lymphocyte of therapeutic group were distinctly higher than those in controlled group. Furthermore, the value of $\mathrm{CD}_{4}{ }^{+} / \mathrm{CD}_{8}{ }^{+}$and the content of IL- 2 and IFN- $\gamma$ were higher after the patients are in the treatment of cordyceps sinensis liposome oral liquid [38]. After using cordyceps sinensis liposome oral liquid for antifibrosis of liver, the authors observed that alanine aminotransferase (ALT), collagen type IV (C-IV), hyaluronic acid, and so on, improved well in the therapeutic group as compared to the controlled group [39].
Recently, combination therapy has drawn great attention as one of the most widely treatment strategies in clinical area. Thus, cordyceps sinensis can exert more synergistic effect with astragali L. and Salvia miltiorrhiza. The liposome oral liquid prepared containing cordyceps sinensis, astragali L. and Salvia miltiorrhiza has a good curative effect in treatment of chronic hepatitis B by improving the liver function, resisting virus, and preventing liver fibrosis [40].

\section{Conclusion}

Liposome-based drug delivery system has been considered as a promising carrier for plant polysaccharides, because it can improve plant polysaccharide stability and bioavailability, enhance the pharmacodynamic action, and induce the target.

\section{Acknowledgments}

The authors thank the National Natural Science Foundation of China for financial support (nos. 81001643, 30901547, 2010DFA32660). This work was also Supported by the Science and Technology Planning Project of Guangdong Province (nos. 2011B037100058, 2011108102020, S2011010005836). This paper was also supported by the Research Fund of the University of Macau (MYRG 208 (Y2-L4)ICMS11-WYT) and UL016/09-Y4/CMS/WYT01/ICMS.

\section{References}

[1] I. A. Schepetkin and M. T. Quinn, "Botanical polysaccharides: macrophage immunomodulation and therapeutic potential," International Immunopharmacology, vol. 6, no. 3, pp. 317-333, 2006.

[2] C. G. O. Wasonga, S. A. Okoth, J. C. Mukuria, and C. O. A. Omwandho, "Mushroom polysaccharide extracts delay progression of carcinogenesis in mice," Journal of Experimental Therapeutics and Oncology, vol. 7, no. 2, pp. 147-152, 2008.

[3] J. Zhang, Y. Yu, Z. Zhang et al., "Effect of polysaccharide from cultured Cordyceps sinensis on immune function and anti-oxidation activity of mice exposed to ${ }^{60} \mathrm{Co}$," International Immunopharmacology, vol. 11, no. 12, pp. 2251-2257, 2011.

[4] S. L. Xiong, A. Li, N. Huang, F. Lu, and D. Hou, "Antioxidant and immunoregulatory activity of different polysaccharide fractions from tuber of Ophiopogon japonicus," Carbohydrate Polymers, vol. 86, no. 3, pp. 1273-1280, 2011.

[5] Y. Sun, H. Liang, G. Cai et al., "Sulfated modification of the water-soluble polysaccharides from Polyporus albicans mycelia and its potential biological activities," International Journal of Biological Macromolecules, vol. 44, no. 1, pp. 14-17, 2009.

[6] Q. Y. Liu, Y. M. Yao, Y. Yu, N. Dong, and Z. Y. Sheng, "Astragalus polysaccharides attenuate postburn sepsis via inhibiting negative immunoregulation of $\mathrm{CD} 4{ }^{+} \mathrm{CD} 25^{\text {high }} \mathrm{T}$ cells," PLoS One, vol. 6, no. 6, Article ID e19811, 2011.

[7] I. A. Shchepetkin, "Activation of macrophages by plant polysaccharides," Antibiotiki i Khimioterapiya, vol. 49, no. 1, pp. 35-42, 2004.

[8] C. X. Dong, K. Hayashi, Y. Mizukoshi et al., "Structures and anti-HSV-2 activities of neutral polysaccharides from an edible plant, Basella rubra L," International Journal of Biological Macromolecules, vol. 50, no. 1, pp. 245-249, 2012. 
[9] X. Chen, W. Nie, G. Yu et al., "Antitumor and immunomodulatory activity of polysaccharides from Sargassum fusiforme," Food and Chemical Toxicology, vol. 50, no. 3-4, pp. 695-700, 2011.

[10] R. Xu, H. Ye, Y. Sun et al., "Preparation, preliminary characterization, antioxidant, hepatoprotective and antitumor activities of polysaccharides from the flowerof tea plant (Camellia sinensis)," Food and Chemical Toxicology. In press.

[11] K. T. Inngjerdingen, S. Meskini, I. Austarheim et al., "Chemical and biological characterization of polysaccharides from wild and cultivated roots of Vernonia kotschyana," Journal of Ethnopharmacology, vol. 139, no. 2, pp. 350-358, 2012.

[12] R. Chang, "Bioactive polysaccharides from traditional chinese medicine herbs as anticancer adjuvants," Journal of Alternative and Complementary Medicine, vol. 8, no. 5, pp. 559-565, 2002.

[13] X. Nie, B. Shi, Y. Ding, and W. Tao, "Preparation of a chemically sulfated polysaccharide derived from Grifola frondosa and its potential biological activities," International Journal of Biological Macromolecules, vol. 39, no. 4-5, pp. 228-233, 2006.

[14] J. Holderness, I. A. Schepetkin, B. Freedman et al., "Polysaccharides isolated from Acai fruit induce innate immune responses," PLoS One, vol. 6, no. 2, Article ID e17301, 2011.

[15] M. Chen, Z. Zhong, W. Tan, S. Wang, and Y. Wang, "Recent advances in nanoparticle formulation of oleanolic acid," Chinese Medicine, vol. 6, article 20, 2011.

[16] F. Li, Y. Zhang, and Z. Zhong, "Antihyperglycemic effect of ganoderma lucidum polysaccharides on streptozotocininduced diabetic mice," International Journal of Molecular Sciences, vol. 12, no. 9, pp. 6135-6145, 2011.

[17] R. Rajera, K. Nagpal, S. K. Singh, and D. N. Mishra, "Niosomes: a controlled and novel drug delivery system," Biological and Pharmaceutical Bulletin, vol. 34, no. 7, pp. 945-953, 2011.

[18] S. Javed, K. Kohli, and M. Ali, "Reassessing bioavailability of silymarin," Alternative Medicine Review, vol. 16, no. 3, pp. 239249, 2011.

[19] M. L. Gonzalez-Rodriguez and A. M. Rabasco, "Charged liposomes as carriers to enhance the permeation through the skin," Expert Opinion on Drug Delivery, vol. 8, no. 7, pp. 857-871, 2011.

[20] L. Ni, H. Caixia, Z. Cheng et al., "Update of novel micropreparations of polysaccharides," Chinese Journal of Modern Applied Pharmacy, vol. 26, no. 8, pp. 620-623, 2009.

[21] F. Zuozhen, G. Dongxiu, and Z. Xiaoting, "Study on the preparation process of the Codyceps sinensis Sacc polysaccharide liposomal oral liquid by orthogonal design," China Pharmaceuticals, vol. 14, no. 8, pp. 50-51, 2005.

[22] F. Yunpeng, H. Deyun, H. Yuanliang et al., "Preparation condition optimization of astragalus polysaccharide liposome by orthogonal test," Chinese Traditional and Herbal Drugs, vol. 42, no. 3, pp. 470-473, 2011.

[23] G. Chuanxin, Y. Hai, and L. Xueli, "Study on the preparation of polysaccharides liposome of Grifola frondosa," China Pharmacy, vol. 21, no. 39, pp. 3687-3689, 2010.

[24] J. H. Wang, R. Yang, Q. N. Fan, K. P. Wang, and M. H. Hu, "Optimizing preparation technique of Polyporus umbellatus polysaccharides long circulating liposomes by orthogonal test," Chinese Traditional and Herbal Drugs, vol. 38, no. 10, pp. 1484-1487, 2007.

[25] W. Kaiping, Z. Yu, and Z. Jun, "Preparation of Polyporus umbellatus polysaccharides long circulating liposomes," Chinese Traditional and Herbal Drugs, vol. 36, no. 3, pp. 368-370, 2005.
[26] G. Franz, "Polysaccharides in pharmacy: current applications and future concepts," Planta Medica, vol. 55, no. 6, pp. 493497, 1989.

[27] W. Gu, Y. Wang, T. Wu et al., "Linear sweep voltammetric studies on the complex of alizarin red $s$ with aloe polysaccharide and determination of aloe polysaccharide," Carbohydrate Research, vol. 349, pp. 82-85, 2012.

[28] Z. Sheng, L. Xiangzhou, W. Zhiping et al., "Research progress on extraction, purification and content determination of plant polysaccharides," Chemistry and Industry of Forest Products, vol. 29, pp. 238-242, 2009.

[29] F. Zuozhen, G. Dongxiu, and Z. Xiaoting, "Quality standards for the Cordyceps sinensis Sacc polysaccharide liposomal oral liquid," Journal of Shenyang Pharmaceutical University, vol. 22, no. 3, pp. 203-206, 2005.

[30] G. Weiying and W. Di, "Relationship of solubility characteristics of Ginsenoside and LBP with liposome entrapment efficiency," China Pharmacy, vol. 22, no. 35, pp. 3300-3302, 2011.

[31] Y. Hongping and Z. Mingyu, "Determination of the entrapment rate of acidic polysaccharide of Spirulina plantensis in liposomes," Chinese Journal of Biochemical Pharmaceutics, vol. 27, no. 4, pp. 225-227, 2006.

[32] S. Lihong, W. Jiansen, L. Ya et al., "Research and application of plant polysaccharide," Chinese Agricultural Science Bulletin, vol. 27, no. 2, pp. 349-352, 2011.

[33] F. Shujie, W. Naiping, and H. Renbin, "Advance in the research on the immunoregulation of Botan-polysaccharides," Lishizhen Medicine and Materia Media Research, vol. 19, no. 1, pp. 99-101, 2008.

[34] C. Yun, P. Yan, C. Bangyin et al., "Comparison of the immune regulatory effect of the lentinan liposome and injection in normal mice," Chinese Journal of Hospital Pharmacy, vol. 29, no. 19, pp. 1624-1627, 2009.

[35] M. Babincova, E. Sustekova, and E. Machova, "Enzymatic digestion of liposome-bound polysaccharides: evidence of bridging mechanism," General Physiology and Biophysics, vol. 19, no. 3, pp. 323-327, 2000.

[36] L. Hongjun, W. Yinglian, and T. Liang, "Effect of Cordyceps polysaccharide liposomes(CPL) on the expression of TGF $\beta 1 \mathrm{mRNA}$ in mice with immune hepatic fibrosis induced by concanaval in A," Journal of Clinical Research, vol. 21, no. 5, pp. 500-503, 2004.

[37] N. Ehrlich, A. L. Christensen, and D. Stamou, "Fluorescence anisotropy based single liposome assay to measure moleculemembrane interactions," Analytical Chemistry, vol. 83, no. 21, pp. 8169-8176, 2011.

[38] S. Rongling, L. Guozhong, and W. Chunjuan, "Effect of compound Cordyceps sinensis Liposome oral liquid on the immunity of Chronic Hepatitis B cell," China Pharmacist, vol. 8, no. 10, pp. 848-849, 2005.

[39] J. Weiling, S. Wanfeng, and S. Rongling, "Clinical observation of compound Cordyceps sinensis liposome oral liquid in treatment of liver fibrosis," Anti-Infection Pharmacy, vol. 4, no. 3, pp. 126-127, 2007.

[40] S. Wanfeng, S. Rongling, and J. Weiling, "Compound Cordyceps sinensis liposome oral liquid in treatment of patients with Chronic Hepatitis B," China Pharmacist, vol. 6, no. 7, pp. 438439, 2003. 

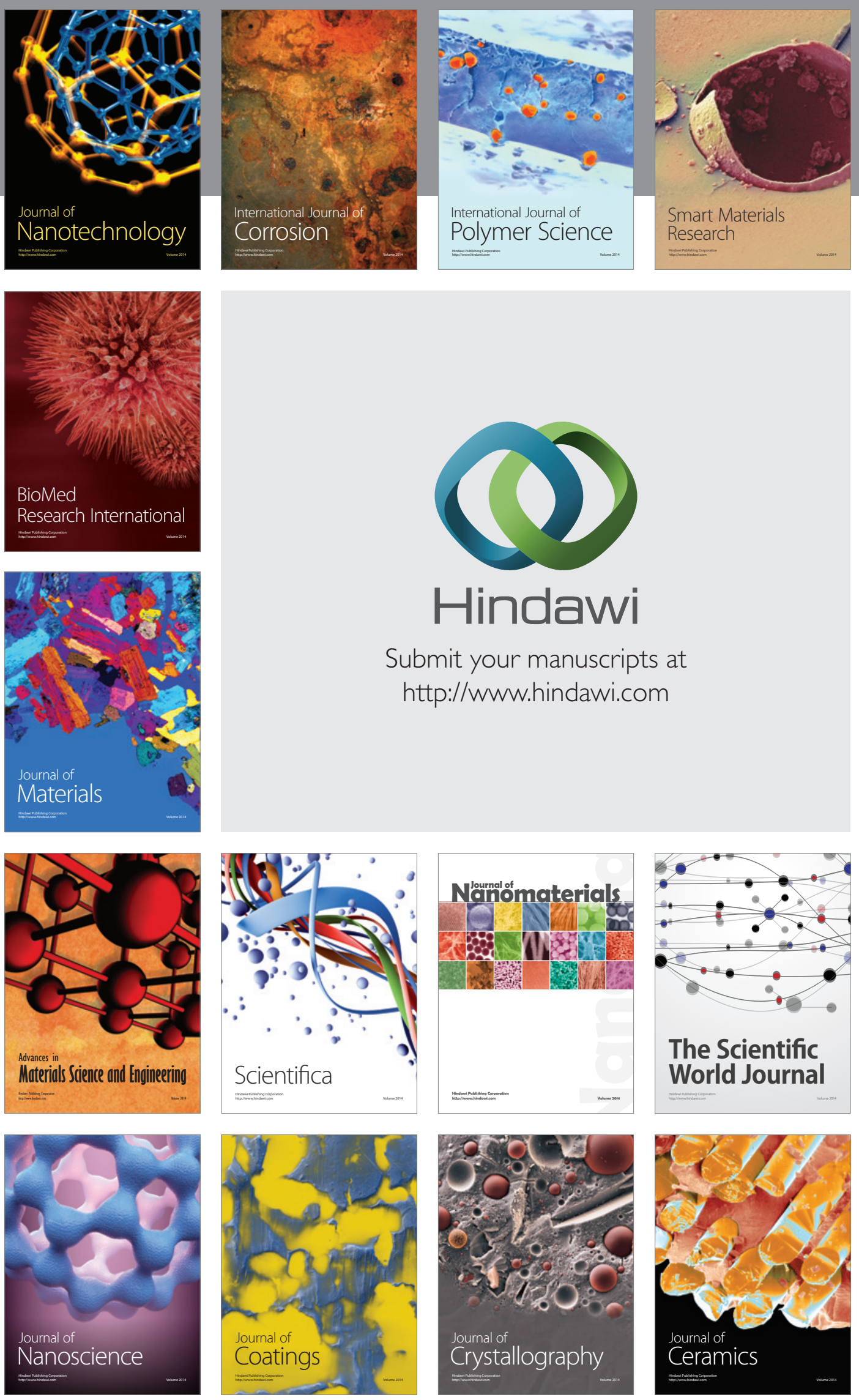

The Scientific World Journal

Submit your manuscripts at

http://www.hindawi.com

\section{World Journal}

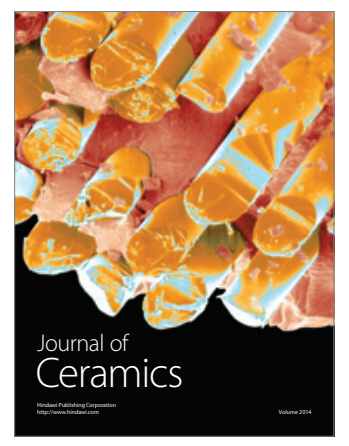

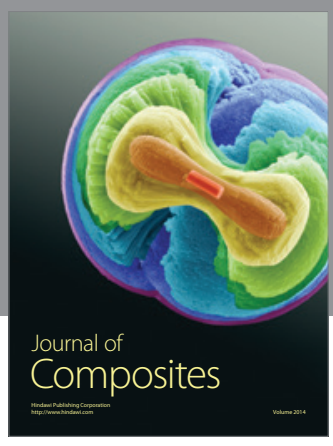
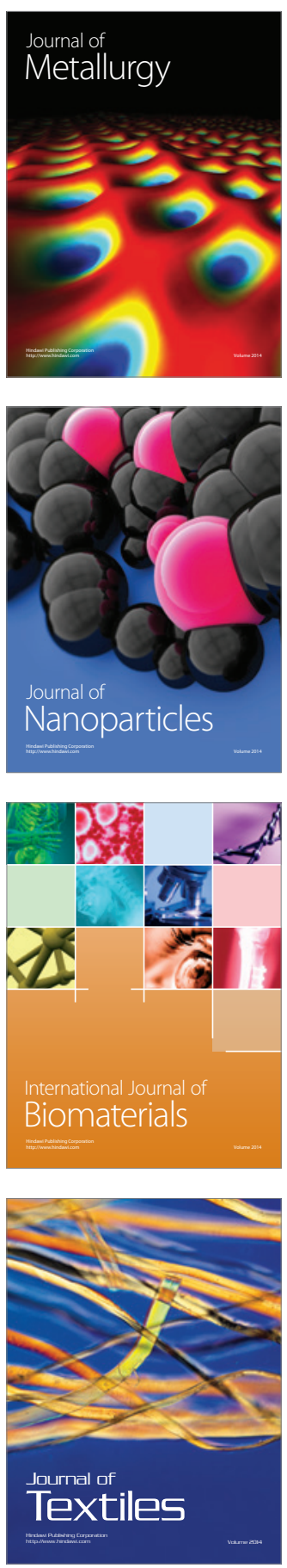